\title{
Association of PIK3CA and MDM2 SNP309 with Cervical Squamous Cell Carcinoma in a Philippine Population
}

\author{
Ourlad Alzeus G Tantengco ${ }^{1,2}$, Yukiko Nakura $^{2}$, Michinobu Yoshimura ${ }^{2}$, Erlidia \\ F Llamas-Clark ${ }^{3}$, Itaru Yanagihara ${ }^{2 *}$
}

\begin{abstract}
Background: PIK3CA and MDM2 SNP309 have been studied to be associated with cervical cancer. PIK3CA mutation is associated with poor treatment response and low survival rate while $M D M 2$ is associated with tumorigenesis and poor prognosis in cervical cancer. Thus, we determined the prevalence of PIK3CA and MDM2 mutations in Filipino cervical cancer patients. Methods: Twenty-eight formalin-fixed paraffin-embedded cervical squamous cell carcinoma and 16 non-malignant cervix tissue biopsies of Filipino patients were subjected to PIK3CA gene and MDM2 SNP309 (rs2279744) analysis. Results: PIK3CA gene was found mutated in three (10.71\%) out of 28 cervical cancer patients included in this study. Among the HPV-negative cervical cancer patients, two $(28.57 \%)$ were positive for PIK3CA mutation and only one (4.76 \%) tested positive among the HPV-positive cervical cancer patients. MDM2 SNP309 analysis revealed that $\mathrm{T}$ allele $(71.43 \%)$ was more common in cervical cancer patients compared to the control group. TG genotype $(\mathrm{p}=0.03 ; \mathrm{OR}=0.18,95 \%$ CI 0.04-0.76) was associated with lower rates of cervical cancer when TT genotype was used as a reference point. Conclusion: PIK3CA gene mutation was present among Filipino cervical cancer patients and not in control patients. MDM2 SNP309 analysis revealed that TG genotype has lower association to cervical cancer when compared with the TT and GG genotypes.
\end{abstract}

Keywords: $M D M 2-P I K 3 C A$ - mutation- HPV- cervical cancer

Asian Pac J Cancer Prev, 20 (7), 2103-2107

\section{Introduction}

Cervical cancer continues to be the second most frequent cancer among Filipino women. It is estimated that almost 6,670 Filipino women are diagnosed with cervical cancer and 2,832 die from this disease annually (ICO/IARC Information Centre on HPV and Cancer, 2017). One of the established necessary risk factors for cervical cancer are oncogenic types of human papillomavirus (HPV). Cells infected by this virus can develop precancerous properties which can lead to cervical intraepithelial neoplasia (CIN) or adenocarcinoma in situ (AIS). Without treatment, CIN grade II/III and AIS can progress to squamous cell carcinoma or adenocarcinoma, respectively (Bosch et al., 1995).

Nucleic acid sequencing is now being used to determine gene mutations that can be used as potential diagnostic or prognostic tools for cancer patients. In cervical cancer patients, mutations in PIK3CA gene was found to be associated with worse overall survival and cancer-specific survival (Lachkar et al., 2018). A study in Chinese population showed that patients with
PIK3CA mutations belong to older populations and postmenopausal patients. It is more common among patients with cervical squamous cell carcinoma and is associated with distant metastases (Xiang et al., 2015). Another study detected PIK3CA gene mutation rate of $11 \%$ in adenocarcinoma, and 5\% in squamous cell carcinoma patients (Tornesello et al., 2014).

Murine double-minute 2 homolog (MDM2) is another gene that has been studied to be associated with tumorigenesis and poor prognosis in several types of cancer including cervical cancer (Zhang et al., 2017). MDM2 single nucleotide polymorphism gene (rs2279744) is in the position 309 in the first intron of the MDM2 oncogene. Replacement of $\mathrm{T}$ with $\mathrm{G}$ allele increased MDM2 mRNA and protein synthesis and decreased the p53 tumor suppressor activity. MDM2 SNP309 (rs2279744) was found to be associated in the development of cervical lesions in women with HPV infection. It might also serve as a marker for assessing the progression low squamous intraepithelial lesion (LSIL) to high squamous intraepithelial lesion (HSIL) (Amaral et al., 2014).

${ }^{1}$ College of Medicine, University of the Philippines Manila, ${ }^{3}$ Department of Obstetrics and Gynecology, College of Medicine, University of the Philippines-Philippines General Hospital, Manila, Philippines ${ }^{2}$ Department of Developmental Medicine, Research Institute, Osaka Women's and Children's Hospital, Izumi, Osaka, Japan. *For Correspondence: itaruy@wch.opho.jp 
Identification of known genetic mutations and polymorphisms in cervical cancer and their association with HPV infection status will help in further understanding the contribution of somatic genomic alteration in the pathogenesis of cervical cancer. Therefore, in this study, we investigated the frequency of PIK3CA and MDM2 mutations in Filipino patients with cervical squamous cell carcinoma.

\section{Materials and Methods}

\section{Patient and tissue samples}

This is a case-control study which included 28 formalin-fixed paraffin-embedded cervical squamous cell carcinoma and 16 non-malignant cervix tissue biopsies of patients seen at the Philippine General Hospital from January to December 2017. Cases and control samples were selected through simple random sampling from the list of cervical cancer patients during 2017 in the Philippine General Hospital. Patients diagnosed with cervical squamous cell carcinoma and with complete clinical data were included in the study. Exclusion criteria include antimicrobial treatment within one month prior to the present study. Matching was not performed in this study. This study was approved by the University of the Philippines Manila Research Ethics Board (UPMREB Registration No.: 2018-016-01).

\section{DNA Extraction}

De-identified formalin fixed paraffin embedded (FFPE) cervical tissues were retrieved from the Section of Surgical Pathology at the Department of Laboratories of the Philippine General Hospital. Approximately, $5 \mu \mathrm{m}$ slices of the FFPE samples were used for total genomic DNA extraction according the protocol of Maxwell ${ }^{\circledR}$ RSC DNA FFPE Kit (Promega, Japan). The concentration of the extracted DNA in aqueous solution was checked using NanoDrop 2000 Spectrophotometer (Thermo Fisher Scientific, Wilmington, DE, USA).

\section{PIK3CA exon 9 mutation analysis}

PIK3CA exon 9 was amplified by PCR using the primers: PIK3-9-F3 (5'-GAGTAACAGACTAGCTAGAGAC-3') with PIK3-9-R2 (5'-ACAGAGAATCTCCATTTTAGC-3'). PCR reactions were performed using $1 \mu \mathrm{L}$ gDNA, $6.25 \mu \mathrm{L}$ $2 \times$ QuantiTect $^{\circledR}$ SYBR $^{\circledR}$ Green PCR Master Mix (Qiagen, Japan), $0.625 \mu \mathrm{L}$ each of $10 \mu \mathrm{M}$ primers and $4 \mu \mathrm{L}$ of deionized distilled water in a total volume of $12.5 \mu \mathrm{L}$. The PCR assay was performed using MJ Research, Chromo 4 system (Reno, Nevada, USA). The PCR was conducted with an initial denaturation of $95^{\circ} \mathrm{C}$ for $15 \mathrm{~min}$, followed by 35 cycles of $95^{\circ} \mathrm{C}$ for $20 \mathrm{sec}$, annealing temperature of $58^{\circ} \mathrm{C}$ for $30 \mathrm{sec}$, extension temperature of $72^{\circ} \mathrm{C}$ for 30 sec, and a final cycle of $72^{\circ} \mathrm{C}$ for $4 \mathrm{~min}$.

\section{MDM2 SNP309 (rs2279744) analysis}

MDM2 gene including the SNP309 (rs2279744) region was amplified using the following primer sequences: forward (5'-CGGGAGTTCAGGGTAAAGGT-3') and reverse (5'-AGCAAGTCGGTGCTTACCTG-3') (Hu et al., 2010). PCR reactions were performed using $1 \mu \mathrm{L}$
gDNA, $6.25 \mu \mathrm{L} 2 \times$ QuantiTect $^{\circledR}$ SYBR $^{\circledR}$ Green PCR Master Mix (Qiagen, Japan), $0.625 \mu \mathrm{L}$ each of $10 \mu \mathrm{M}$ primers and $4 \mu \mathrm{L}$ of deionized distilled water in a total volume of $12.5 \mu \mathrm{L}$. The assays were performed using MJ Research, Chromo 4 system (Reno, Nevada, USA). The PCR was conducted with an initial denaturation of $95^{\circ} \mathrm{C}$ for $15 \mathrm{~min}$, followed by 35 cycles of $95^{\circ} \mathrm{C}$ for 20 sec, annealing temperature of $63^{\circ} \mathrm{C}$ for $30 \mathrm{sec}$, extension temperature of $72^{\circ} \mathrm{C}$ for $30 \mathrm{sec}$, and a final cycle of 72 ${ }^{\circ} \mathrm{C}$ for $4 \mathrm{~min}$.

\section{Nucleic acid sequencing}

All PCR amplicons of PIK3CA and MDM2 genes were purified using NucleoSpin ${ }^{\circledR}$ Gel and PCR Clean Up (Machery-Nagel, Germany) and were subjected to DNA nucleotide sequencing using the Big Dye ${ }^{\mathrm{TM}}$ Terminator v3.1 Cycle Sequencing Kit on an ABI 3130 Genetic Analyzer (Thermo Fisher Scientific, MA, 220 USA) according to the manufacturer's instructions.

\section{Statistical Analysis}

Fisher exact test and t-test were calculated using Open Epi Software (https://www.openepi.com/Menu/ OE_Menu.htm). Allele frequencies were determined by the direct count of the alleles. Genotypic distributions were examined for significant departure from Hardy-Weinberg equilibrium by Chi square-test. SNPStat online software (https://www.snpstats.net/start.htm) was used to analyze the association of the allele and genotype frequencies with cervical cancer. Association was considered statistically significant when $\mathrm{p}$ value was less than 0.05 .

\section{Results}

This study included 28 cases of cervical cancer and 21 controls with non-malignant cervix from the Philippine General Hospital. The mean age of the cervical cancer patients was $52.75 \pm 13.16$ years while the control patients was $47.31 \pm 15.02$ years. Patients in the case group were significantly older compared to patients in the control group.

\section{PIK3CA gene mutation analysis}

All cervical cancer patients were analyzed for PIK3CA mutation (Table 1). Based on our previous study, 21 cervical cancer were HPV-positive and 7 were HPV-negative. The exon 9 of PIK3CA gene was found mutated in three $(10.71 \%)$ out of 28 cervical cancer patients included in this study (Table 2). Two patients have $E 545 \mathrm{~K}$ mutation while one has $E 545 \mathrm{~A}$ mutation. All three $P I K 3 C A$ gene mutants were from patients with FIGO stage 3 cervical squamous cell carcinoma. Among HPV-negative cervical cancer patients, two (28.57\%) were positive for PIK3CA mutation while among HPV-positive cervical cancer patients, only one $(4.76 \%)$ tested positive for PIK3CA mutation. This mutation was not found among the control patients.

\section{MDM2 SNP309 (rs2279744) analysis}

The frequencies of each allele and genotype were shown in Table 3. MDM2 polymorphism analysis 
Table 1. Histological Characteristics of Cervical Cancer Samples, HPV status, PIK3CA (exon 9) Mutations and MDM2 Single Nucleotide Polymorphisms

\begin{tabular}{|c|c|c|c|c|c|}
\hline Patient No & Histology & FIGO Staging & HPV Status & $P I K 3 C A$ mutation & MDM2 SNP309 \\
\hline p1 & Cervical SCCA, nonkeratinizing & $3 \mathrm{~B}$ & Negative & & $\mathrm{T} / \mathrm{T}$ \\
\hline p2 & Cervical SCCA, nonkeratinizing & $3 \mathrm{~B}$ & HPV 16 & & $\mathrm{~T} / \mathrm{G}$ \\
\hline p3 & Cervical SCCA, nonkeratinizing & $3 \mathrm{~B}$ & HPV 16 & & $\mathrm{~T} / \mathrm{T}$ \\
\hline $\mathrm{p} 4$ & Cervical SCCA, nonkeratinizing & $3 \mathrm{~B}$ & HPV 16, 18 & & $\mathrm{~T} / \mathrm{T}$ \\
\hline p5 & Cervical SCCA, nonkeratinizing & $3 \mathrm{~B}$ & Negative & & $\mathrm{T} / \mathrm{T}$ \\
\hline p6 & Cervical SCCA, nonkeratinizing & $3 \mathrm{~B}$ & HPV 16 & & $\mathrm{~T} / \mathrm{T}$ \\
\hline p7 & Cervical SCCA, nonkeratinizing & $4 \mathrm{~A}$ & HPV 52 & & $\mathrm{~T} / \mathrm{T}$ \\
\hline p8 & Cervical SCCA, nonkeratinizing & $3 \mathrm{~B}$ & HPV 18 & & $\mathrm{~T} / \mathrm{G}$ \\
\hline p9 & Cervical SCCA, nonkeratinizing & $2 \mathrm{~B}$ & HPV 16, 52 & & $\mathrm{G} / \mathrm{G}$ \\
\hline p10 & Cervical SCCA, nonkeratinizing & $3 \mathrm{~B}$ & HPV 16, 18 & & $\mathrm{~T} / \mathrm{T}$ \\
\hline p11 & Cervical SCCA, nonkeratinizing & $3 \mathrm{~B}$ & HPV 16 & & $\mathrm{~T} / \mathrm{T}$ \\
\hline p12 & Cervical SCCA, nonkeratinizing & $3 \mathrm{~B}$ & HPV 52 & & $\mathrm{~T} / \mathrm{G}$ \\
\hline p13 & Cervical SCCA, nonkeratinizing & $3 \mathrm{~B}$ & Negative & & $\mathrm{T} / \mathrm{T}$ \\
\hline p14 & Cervical SCCA, nonkeratinizing & $3 \mathrm{~B}$ & HPV 52 & & $\mathrm{~T} / \mathrm{G}$ \\
\hline p15 & Cervical SCCA, nonkeratinizing & $2 \mathrm{~B}$ & HPV 16 & & $\mathrm{~T} / \mathrm{T}$ \\
\hline p16 & Cervical SCCA, nonkeratinizing & $3 \mathrm{~B}$ & HPV 18 & E545K & $\mathrm{T} / \mathrm{T}$ \\
\hline p17 & Cervical SCCA, nonkeratinizing & $3 \mathrm{~A}$ & HPV 16 & & $\mathrm{~T} / \mathrm{T}$ \\
\hline $\mathrm{p} 18$ & Cervical SCCA, nonkeratinizing & $3 \mathrm{~B}$ & HPV 52 & & TG \\
\hline p19 & Cervical SCCA, nonkeratinizing & $3 \mathrm{~B}$ & Negative & E545K & $\mathrm{G} / \mathrm{G}$ \\
\hline p20 & Cervical SCCA, nonkeratinizing & $3 \mathrm{~B}$ & Negative & E545A & $\mathrm{G} / \mathrm{G}$ \\
\hline p21 & Cervical SCCA, nonkeratinizing & $3 \mathrm{~B}$ & HPV 52 & & $\mathrm{~T} / \mathrm{G}$ \\
\hline p22 & Cervical SCCA, nonkeratinizing & $3 \mathrm{~B}$ & Negative & & $\mathrm{G} / \mathrm{G}$ \\
\hline p23 & Cervical SCCA, nonkeratinizing & $3 \mathrm{~B}$ & HPV 18 & & $\mathrm{~T} / \mathrm{T}$ \\
\hline p24 & Cervical SCCA, nonkeratinizing & $3 \mathrm{~B}$ & HPV 16 & & $\mathrm{~T} / \mathrm{T}$ \\
\hline p25 & Cervical SCCA, nonkeratinizing & 1B1 & HPV 16 & & $\mathrm{~T} / \mathrm{T}$ \\
\hline p26 & Cervical SCCA, nonkeratinizing & $3 \mathrm{~B}$ & HPV 18 & & $\mathrm{~T} / \mathrm{T}$ \\
\hline p27 & Cervical SCCA, nonkeratinizing & $3 \mathrm{~B}$ & Negative & & $\mathrm{T} / \mathrm{G}$ \\
\hline p28 & Cervical SCCA, nonkeratinizing & $3 \mathrm{~B}$ & HPV 16 & & $\mathrm{~T} / \mathrm{G}$ \\
\hline
\end{tabular}

Table 2. PIK3CA Mutation and Clinical Characteristics of Cervical Squamous Cell Carcinoma Patients ( $\mathrm{n}=28$ )

\begin{tabular}{|c|c|c|c|}
\hline & PIK3CA wild type $(\mathrm{n}=25)$ & PIK3CA mutant $(n=3)$ & $\mathrm{p}$ values \\
\hline Mean age \pm SD & $51.33 \pm 14.46$ & $48.33 \pm 11.72$ & $\mathrm{p}=0.71^{*}$ \\
\hline FIGO Staging & & & $\mathrm{p}>0.99^{\dagger}$ \\
\hline $\mathrm{I}(\mathrm{n}=1)$ & 1 & 0 & \\
\hline II $(n=2)$ & 2 & 0 & \\
\hline III $(n=24)$ & 21 & 3 & \\
\hline $\operatorname{IV}(n=1)$ & 1 & 0 & \\
\hline HPV Status & & & $\mathrm{p}=0.29^{\dagger}$ \\
\hline Negative $(n=7)$ & $5(71.43 \%)$ & $2(28.57 \%)$ & \\
\hline Positive $(n=21)$ & $20(95.24 \%)$ & $1(4.76 \%)$ & \\
\hline
\end{tabular}

revealed that $\mathrm{T}$ allele $(71.43 \%)$ was more common in cervical cancer patients compared to the control group. The G allele for MDM2 SNP309 did not show significant association with cervical cancer. The SNP was evaluated in compliance with the Hardy-Weinberg principle. The difference in the genotypes was significant with regards to the TG genotype when TT was used as a reference point $(p=0.03)$. Surprisingly, the TG heterozygous genotype provided protection [OR $=0.18,95 \%$ CI $0.04-0.76$ ] against cervical cancer when compared to the TT homozygous genotype. There was no significant difference in the distribution of the TT homozygous genotype in comparison with the GG homozygous state. Using the overdominant model of inheritance, TG genotype still showed lower association [OR $=0.18,95 \%$ CI $0.05-0.69]$ with cervical cancer when compared to the TT and GG 
Table 3. HPV Status and Allele Frequency of MDM2 SNP309 (rs2279744) in Patients with Cervical Cancer (n=28) and Normal Cervix $(\mathrm{n}=16)$

\begin{tabular}{lccccc}
\hline HPV Status & Alleles & Cases & Control & OR (95\% CI) & p values \\
\hline HPV Positive Samples & T & $33(79.00 \%)$ & $4(50.00 \%)$ & 1.00 & 0.10 \\
& G & $9(21.00 \%)$ & $4(50.00 \%)$ & $0.27(0.06-1.31)$ & \\
HPV Negative Samples & T & $7(50.00 \%)$ & $15(62.00 \%)$ & 1.00 & 0.45 \\
All Samples & G & $7(50.00 \%)$ & $9(38.00 \%)$ & $1.67(0.44-6.33)$ & \\
& $\mathrm{T}$ & $40(71.43 \%)$ & $19(59.38 \%)$ & 1.00 & 0.25 \\
\hline
\end{tabular}

Table 4. HPV Status and Genotype Frequency of MDM2 SNP309 (rs2279744) in Patients with Cervical Cancer $(\mathrm{n}=28)$ and Normal Cervix $(\mathrm{n}=16)$

\begin{tabular}{lccccc}
\hline HPV Status & Genotypes & Cases & Control & OR (95\% CI) & $\mathrm{p}$ values \\
\hline HPV Positive Samples & TT & $13(61.90 \%)$ & $0(0 \%)$ & 1.00 & $\mathrm{P}=0.02$ \\
& TG & $7(33.30 \%)$ & $4(100 \%)$ & 0.00 & 1.00 \\
HPV Negative Samples & GG & $1(4.80 \%)$ & $0(0 \%)$ & 1.00 & $\mathrm{P}=0.09$ \\
& TT & $3(42.90 \%)$ & $4(33.33 \%)$ & $0.19(0.01-2.50)$ & \\
All Samples & TG & $1(14.30 \%)$ & $7(58.33 \%)$ & $4.00(0.27-60.33)$ & $\mathrm{p}=0.03$ \\
& GG & $3(42.90 \%)$ & $1(8.33 \%)$ & 1.00 & \\
& TT & $16(57.14 \%)$ & $4(25.00 \%)$ & $18(0.04-0.76)$ & $1.00(0.09-11.59)$ \\
\hline
\end{tabular}

homozygous genotypes.

When HPV status was considered, it was observed that TG genotype was still protective against cervical cancer among HPV positive patients (Table 4). TT and GG homozygous genotypes have the same risk for cervical cancer. Among HPV negative patients, it was shown that GG genotype has 4 times higher OR for cervical cancer compared to the TT genotype, however, the difference was not statistically significant.

\section{Discussion}

In this study, we determine the frequency of PIK3CA and MDM2 SNP309 (rs2279744) in Filipino cervical cancer patients. At this stage, there is still no clear association between the presence of PIK3CA mutation and $M D M 2$ polymorphisms and the occurrence of cervical cancer. There are few studies in this area and the conclusions remain inconclusive. These genetic mutation and polymorphisms have not been studied for cervical cancer patients in the Philippines. This was the first study on Filipino patients.

In this study, PIK3CA mutation (10.71\%) was only found among cervical cancer patients. This is consistent with the study conducted among Chinese population where $13.6 \%$ (105 of 771) of patients with resected cervical cancer harbored non-synonymous PIK3CA mutations (Xiang et al., 2015). One previous study about genetic alterations in cervical cancer patients determined that PIK3CA has the highest gene mutation rates among all other cancer-associated genes included in their study. PIK3CA mutation rate was as high as $62.5 \%$ among American Indian population (Femi, 2018). Another study conducted in Italy revealed a 5\% prevalence of PIK3CA mutations among patient with cervical squamous cell carcinoma (Tornesello et al., 2014). These studies show the possible association between PIK3CA mutations and the occurrence of cervical cancer.

This study showed that E545K was the most common site of mutation in PIK3CA gene. This is similar to the results of previous studies that $\mathrm{E} 545 \mathrm{~K}$ is the most predominant site of mutation in the helical domain of the PIK3CA gene (Akbarov et al., 2017; Lou et al., 2015; Xiang et al., 2015). This missense mutation is associated with increased enzymatic activity independent of upstream signaling which results to stimulation of signalling through the Akt pathway thereby increasing cell invasion and metastasis (Arjumand et al., 2016).

HPV genotyping was previously done for all patients included in this study. This mutation was more common among HPV-negative cervical cancer samples $(28.57 \%)$ than in HPV-positive cervical cancer samples (4.76\%). However, the difference was not statistically significant. A previous study found no correlation between HPV infection and PIK3CA status. However, PIK3CA mutation positivity rate was relatively low among HPV-positive cervical cancer patients (Cui et al., 2009). These findings support the possibility that the PIK3CA mutation and HPV infection may act independently in cervical carcinogenesis.

In this study, showed that GG genotype was associated with cervical cancer regardless of the HPV status of the patient. This parallels the results of previous studies that GG genotype increases the risk of cervical cancer. It is also significantly associated with lymph node metastasis in cervical cancer (Guo et al., 2016; Jianget al., 2010; Singhal et al., 2013).

We also observed that MDM2 SNP309 T allele was 
more common that $\mathrm{G}$ allele in cervical cancer patients. The TT and GG homozygous genotypes have higher risk of cervical cancer compared to the TG genotype. The TG genotype seems to have a protective role against cervical cancer. However, in previous studies on cervical cancer, the $\mathrm{G}$ allele and $\mathrm{TG}$ genotype have higher risk of developing cervical cancer compared to the TT genotype. In other types of cancer such as oropharyngeal carcinoma, MDM2 SNP309 TT genotype has higher association with tumor recurrence when compared to TG and GG genotypes (Guo et al., 2016; Jiang et al., 2010; Singhal et al., 2013; Zhang et al., 2017).

It should be noted that there were also previous studies which showed no significant association between MDM2 SNP309 and cervical cancer. The allele or genotype frequency are the same between cervical cancer patients and healthy control patients (Hu et al., 2010; Meissner et al., 2007; Zhang et al., 2017). These differences regarding the role of MDM2 SNP309 in cervical cancer may be due to the racial differences. Hence, further studies are needed to confirm whether ethnicity can affect the distribution of MDM2 SNP309.

The limitations of this study include the small sample size and the inclusion of only one histological type of cervical cancer. Comparison of the different histological types of cervical cancer may also be warranted for future studies to determine whether the frequency of PIK3CA mutation and MDM2 polymorphism differ based on the histological types of cervical cancer. Future cohort studies with large sample size are needed to establish the association of these genes in cervical cancer.

\section{Acknowledgements}

This work was supported by research grants from the UPM NIH, Grant Number NIH2017043 (O. A. G. T. and E. F. L.); JSPS KAKENHI, Grant Numbers JP17H04237 (I. Y.), JP17K10201 (F. N.) and JP18K16788 (M. Y.); the Japan Agency for Medical Research and Development (AMED), JP17fk0108210 and JPfk0108010 (I. Y.).

\section{References}

Akbarov K, Isayev I, Melikova L, et al (2017). PIK3CA gene mutation frequency among cervical cancer patients in Azerbaijan. Ann Oncol, 28, 86-93.

Amaral CMM, Cetkovská K, Gurgel APAD, et al (2014). MDM2 polymorphism associated with the development of cervical lesions in women infected with Human papillomavirus and using of oral contraceptives. Infect Agent Cancer, 9, 1-8.

Arjumand W, Merry CD, Wang C, et al (2016). Phosphatidyl inositol-3 kinase ( PIK3CA ) E545K mutation confers cisplatin resistance and a migratory phenotype in cervical cancer cells. Oncotarget, 7, 82424-39.

Bosch FX, Manos MM, Muñoz N, et al (1995). Prevalence of human papillomavirus in cervical cancer: A worldwide perspective. J Natl Cancer Inst, 87, 796-802.

Cui B, Zheng B, Zhang XI, Stendahl ULF (2009). Mutation of $P I K 3 C A$ : Possible risk factor for cervical carcinogenesis in older women. Int $J$ Oncol, 34, 409-16.

Femi OF (2018). Genetic alterations and PIK3CA gene mutations and amplifications analysis in cervical cancer by racial groups in the United States. Int J Health Sci (Qassim), 12,
28-32.

Guo H, Yang S, Ding Z, Xu L, Li D (2016). Association between SNP309 promoter polymorphism in the MDM2 and cervical cancer in South-Central China. J Bionanoscience, 10, 501-5.

Hu X, Zhang Z, Ma D, Huettner PC, Massad LS (2010). TP53, $M D M 2, \mathrm{NQO1}$, and susceptibility to cervical cancer. cancer, Epidemiol Biomarkers Prev, 6, 755-62.

ICO/IARC Information Centre on HPV and Cancer (2017). Philippines Human Papillomavirus and Related Cancers, Fact Sheet 2017 (Vol. 2017). (Barcelona, Spain).

Jiang P, Liu J, Zeng X, Li W, Tang J (2010). MDM2 gene promoter polymorphism and risk of cervical cancer in Chinese population. 4th Int Conf Bioinforma Biomed Eng, pp 1-4.

Lachkar B, Minaguchi T, Akiyama A, et al (2018). Prognostic significance of PIK3CA mutation in stage IIB to IVA cervical cancers treated by concurrent chemoradiotherapy with weekly cisplatin. Medicine (Baltimore), 97, e11392.

Lou H, Villagran G, Boland JF, et al (2015). Genome analysis of Latin American cervical cancer: Frequent activation of the PIK3CA pathway. Clin Cancer Res, 21, 5360-70.

Meissner R de V, Barbosa RNF, Fernandes JV, et al (2007). No association between SNP309 promoter polymorphism in the $M D M 2$ and cervical cancer in a study from northeastern Brazil. Cancer Detect Prev, 31, 371-4.

Singhal P, Hussain S, Thakur N, et al (2013). Association of MDM2 and p53 Polymorphisms with the Advancement of Cervical Carcinoma. DNA Cell Biol, 32, 19-27.

Tornesello ML, Annunziata C, Buonaguro L, et al (2014). TP53 and $P I K 3 C A$ gene mutations in adenocarcinoma, squamous cell carcinoma and high-grade intraepithelial neoplasia of the cervix. J Transl Med, 12, 255.

Xiang L, Jiang W, Li J, et al (2015). PIK3CA mutation analysis in Chinese patients with surgically resected cervical cancer. Sci Rep, 5, 1-7.

Zhang J, Zhang Y, Zhang Z (2017). Association of rs2279744 and $r s 117039649$ promoter polymorphism with the risk of gynecological cancer. Medicine (Baltimore), 97, e9554.

Zhang Y, Sturgis EM, Li Y, et al (2017). Modifying effect of mouse double minute-2 promoter variants on risk of recurrence for patients with squamous cell carcinoma of oropharynx. Sci Rep, 7, 39765.

\section{(c) (i) (8)}

This work is licensed under a Creative Commons AttributionNon Commercial 4.0 International License. 